PROCEEDINGS OF THE

AMERICAN MATHEMATICAL SOCIETY

Volume 126, Number 7, July 1998, Pages 2013-2023

S 0002-9939(98)04266-X

\title{
COMPOSITION OPERATORS ON WEIGHTED DIRICHLET SPACES
}

\author{
NINA ZORBOSKA
}

(Communicated by Theodore W. Gamelin)

\begin{abstract}
We characterize bounded and compact composition operators on weighted Dirichlet spaces. The method involves integral averages of the determining function for the operator, and the connection between composition operators on Dirichlet spaces and Toeplitz operators on Bergman spaces. We also present several examples and counter-examples that point out the borderlines of the result and its connections to other themes.
\end{abstract}

\section{INTRODUCTION}

For an analytic function $\phi$ that maps the unit disc $\mathbb{D}$ into itself, we define a composition operator $C_{\phi}$ by

$$
C_{\phi} f=f \circ \phi
$$

for $f$ analytic on $\mathbb{D}$. Composition operators can act on various spaces of functions analytic on $\mathbb{D}$. In each case the main goal is to discover the connection between the properties of the inducing functions $\phi$ and the operator theoretic properties of $C_{\phi}$. References for most of the known results on the subject can be found in [10] and [1].

In this paper we shall determine the functions $\phi$ that induce bounded or compact composition operators on the weighted Dirichlet spaces. Postponing the necessary definitions to Section 2, we give the statement of the result below.

Theorem 1. Let $-1<\alpha<1$ and let $\phi$ belong to the weighted Dirichlet space $D_{\alpha}$. Then:

(a) $C_{\phi}$ is bounded on $D_{\alpha}$ if and only if there exists $\delta>0$ such that

$$
\sup _{a \in \mathbb{D}} \frac{1}{m(D(a, \delta))} \int_{D(a, \delta)} \tau_{\phi, \alpha}(w) d m(w)<\infty ;
$$

(b) $C_{\phi}$ is compact on $D_{\alpha}$ if and only if

$$
\lim _{a \mapsto \partial \mathbb{D}} \frac{1}{m(D(a, \delta))} \int_{D(a, \delta)} \tau_{\phi, \alpha}(w) d m(w)=0,
$$

where $D(a, \delta)$ is a pseudohyperbolic disk and $\tau_{\phi, \alpha}$ is the determining function for $C_{\phi}$ on $D_{\alpha}$.

Received by the editors March 13, 1996 and, in revised form, December 10, 1996.

1991 Mathematics Subject Classification. Primary 47B38; Secondary 30H05.

Key words and phrases. Composition operators, Dirichlet spaces, Carleson measures, angular derivatives.

The author was supported in part by an NSERC grant. 
In the case $\alpha=0$ the result is fairly simple and has been around for a while ([11]). It was not known though if there is a better, pointwise characterization (to be explained in detail later). We give an example showing that our result is the best possible in this case.

The proof of the general result combines the use of the Nevanlinna function as developed in [9] and the results on Toeplitz operators on Bergman spaces from [7]. A similar approach has also been used in the cases of classical Hardy spaces $H^{2}$ and weighted Bergman spaces to determine the composition operators in Schatten p-class ([4]) and to determine composition operators with closed range ([13]).

The result provides a connection between the operator theoretic properties of composition operators (such as boundedness and compactness) and the function theoretic properties of the inducing map.The effect of the theorem on the geometry of the inducing map is illustrated in the last section where we discuss the ramification of the result and construct several examples that lead us to connections to other themes such as angular derivatives, multiplicity of analytic maps and Holder domains.

\section{NOTATION AND BACKGROUND}

We first recall the definition of weighted Dirichlet spaces $D_{\alpha}$ for $-1<\alpha<1$. A function $f$ that is analytic on $\mathbb{D}$ belongs to $D_{\alpha}$ if and only if

$$
\int_{\mathbb{D}}\left|f^{\prime}(z)\right|^{2}(1-|z|)^{\alpha} d m(z)<\infty
$$

where $m$ stands for the normalized Lebesgue area measure of the unit disc $\mathbb{D}$. The norm on $D_{\alpha}$ is defined by

$$
\|f\|_{\alpha}^{2}=|f(0)|^{2}+\int_{\mathbb{D}}\left|f^{\prime}(z)\right|^{2}(1-|z|)^{\alpha} d m(z) .
$$

Note that $D_{0}=\mathcal{D}$, the Dirichlet space. For $\alpha=1$ we obtain $D_{1}=H^{2}$, since the new norm is equivalent to the usual $H^{2}$ norm.

It follows from the closed graph theorem that whenever $\phi$ is not in $D_{\alpha}$, the operator $C_{\phi}$ is not bounded on $D_{\alpha}$. So, the following important observation holds:

- A necessary condition for $C_{\phi}$ to be bounded is that $\phi$ belongs to $D_{\alpha}$.

Thus, a proper question to ask is: which functions $\phi$ from $D_{\alpha}$ will induce bounded operators $C_{\phi}$ on $D_{\alpha}$ ?

The integral part of the $D_{\alpha}$ norm of $C_{\phi} f$ can be transformed by the following use of the change of variable formula:

$$
\begin{gathered}
\int_{\mathbb{D}}\left|f^{\prime}(\phi(z))\right|^{2}\left|\phi^{\prime}(z)\right|^{2}(1-|z|)^{\alpha} d m(z) \\
=\int_{\phi}(\mathbf{D})\left|f^{\prime}(w)\right|^{2} \sum_{j} \frac{\left(1-\left|z_{j}(w)\right|\right)^{\alpha}}{(1-|w|)^{\alpha}}(1-|w|)^{\alpha} d m(w),
\end{gathered}
$$

where $w=\phi(z)$ and $\left\{z_{j}(w)\right\}$ is the set of all (at most countably many) preimages of $w$.

Define

$$
\tau_{\phi, \alpha}(w)=\frac{\sum_{j}\left(1-\left|z_{j}(w)\right|\right)^{\alpha}}{(1-|w|)^{\alpha}}
$$

for $w$ in $\phi(\mathbb{D})$ and set $\tau_{\phi, \alpha}(w)=0$ for $w \notin \phi(\mathbb{D})$. We call $\tau_{\phi, \alpha}$ the determining function for $C_{\phi}$ on $D_{\alpha}$. 
It is clear from the above equation that whenever $\tau_{\phi, \alpha}(w)$ is bounded on $\mathbb{D}$, the operator $C_{\phi}$ is bounded on $D_{\alpha}$.

Remark 1. In general the notation $a \sim b$ ( $a$ equivalent to $b)$ means that there are positive constants $c_{1}$ and $c_{2}$ such that $c_{1} b \leq a \leq c_{2} b$.

For $|z|$ close to 1 , we have that $1-|z|$ is equivalent to $-\log |z|$, and for $|w|$ close to 1 the function $\tau_{\phi, \alpha}(w)$ behaves similarly (in the context that we need) as the function $\sigma_{\alpha}(w)=\sum_{j}\left(-\log \left|z_{j}(w)\right|\right)^{\alpha} /(-\log |w|)^{\alpha}$, where $\sum_{j}\left(-\log \left|z_{j}(w)\right|\right)^{\alpha}$ is the generalized Nevanlinna counting function $N_{\phi, \alpha}(w)$ defined in [8]. The fact that the boundedness of $\sigma_{\alpha}$ is a sufficient condition for the boundedness of $C_{\phi}$ on $D_{\alpha}$ also appears in $[9]$.

Remark 2. The determining function $\tau_{\phi, \alpha}$ is a positive, measurable function, and it is not always bounded when $\alpha<1$. For example, if $\alpha=0$, then $\tau_{\phi, \alpha}(w)=n_{\phi}(w)$, where $n_{\phi}(w)$ is the multiplicity of $\phi$ at $w$. As long as $\phi$ is of unbounded multiplicity, we have that $\tau_{\phi, \alpha}(w) \mapsto \infty$ as $|w| \mapsto 1$. It is true though that $\tau_{\phi, \alpha}(w)$ is in $L^{1}\left((1-|w|)^{\alpha} d m\right)$ for $\phi \in D_{\alpha}$, since

$$
\begin{gathered}
\|\phi\|_{\alpha}^{2}=|\phi(0)|^{2}+\int_{\mathbb{D}}\left|\phi^{\prime}(z)\right|^{2}(1-|z|)^{\alpha} d m(z) \\
=|\phi(0)|^{2}+\int_{\phi(\mathbb{D})} \frac{\sum_{j}\left(1-\left|z_{j}(w)\right|\right)^{\alpha}}{(1-|w|)^{\alpha}}(1-|w|)^{\alpha} d m(w) \\
=|\phi(0)|^{2}+\int_{\mathbb{D}} \tau_{\phi, \alpha}(w)(1-|w|)^{\alpha} d m(w) .
\end{gathered}
$$

For $\alpha>-1$, let $m_{\alpha}$ be a probability measure on $\mathbb{D}$ defined by

$$
d m_{\alpha}(z)=(\alpha+1)(\alpha+2)(1-|z|)^{\alpha} d m(z) .
$$

A weighted Bergman space $A_{\alpha}$ (sometimes denoted by $L_{a}^{2}\left(d m_{\alpha}\right)$ ) is the subspace of $L^{2}\left(\mathbb{D}, d m_{\alpha}\right)$ consisting of functions analytic on $\mathbb{D}$. Note that an analytic function $f$ belongs to the weighted Dirchlet space $D_{\alpha}$ if and only if its derivative belongs to $A_{\alpha}$.

Given a function $\tau$ in $L^{\infty}\left(d m_{\alpha}\right)$, the Toeplitz operator $T_{\tau}$ on $A_{\alpha}$ is defined by $T_{\tau} f=P_{\alpha}(\tau f)$, where $P_{\alpha}$ is the orthogonal projection from $L^{2}\left(d m_{\alpha}\right)$ onto $L_{a}^{2}\left(d m_{\alpha}\right)$. Toeplitz operators with symbols $\tau$ in $L^{1}\left(d m_{\alpha}\right)$ (where $\tau$ is not necessarily bounded) are defined in [3] by the formula

$$
T_{\tau} f(z)=\int_{\mathbb{D}} f(w) \tau(w)(1-w \bar{z})^{1-\alpha} d m_{\alpha}(w),
$$

where $f$ is in $A_{\alpha}$, and $\alpha>-1$. When $f$ and $g$ are polynomials, it follows that

$$
\left\langle T_{\tau} f, g\right\rangle_{A_{\alpha}}=\int f(z) \overline{g(z)} \tau(z) d m_{\alpha}(z) .
$$

The operator $T_{\tau}$ is not always bounded. Its boundedness and compactness are determined by the Carleson type conditions in $([7])$ which we will state later in section 3 .

There is a close connection between Toeplitz operators on Bergman spaces and composition operators on Dirichlet spaces (see [3]). For $f$ and $g$ polynomials and 


$$
\begin{aligned}
& -1<\alpha<1, \\
& \left\langle C_{\phi}^{*} C_{\phi} f, g\right\rangle_{D_{\alpha}}=\int_{\mathbb{D}}(f \circ \phi)^{\prime}(z) \overline{(g \circ \phi)^{\prime}(z)}(1-|z|)^{\alpha} d m(z)+f(\phi(0)) \cdot \overline{g(\phi(0))} \\
& \quad=\int f^{\prime}(w) \overline{g^{\prime}(w)} \cdot \sum \frac{\left(1-\left|z_{j}(w)\right|\right)^{\alpha}}{(1-|w|)^{\alpha}}(1-|w|)^{\alpha} d m(w)+f(\phi(0)) \cdot \overline{g(\phi(0))} \\
& \quad=\int f^{\prime} \overline{g^{\prime}} \tau_{\phi, \alpha} d m_{\alpha}+f(\phi(0)) \cdot \overline{g(\phi(0))}=\left\langle T_{\tau_{\phi, \alpha}} f^{\prime}, g^{\prime}\right\rangle_{A_{\alpha}}+f(\phi(0)) \cdot \overline{g(\phi(0))} .
\end{aligned}
$$

Let $F$ be the differentiation operator from $D_{\alpha}^{0}$ onto $A_{\alpha}$, where $D_{\alpha}^{0}=\{f \in$ $\left.D_{\alpha} ; f(0)=0\right\}$. If $\phi(0)=0$, then $D_{\alpha}^{0}$ is an invariant subspace for $C_{\phi}$. If in addition one of the operators $C_{\phi}$ and $T_{\tau_{\phi, \alpha}}$ is bounded, the above equalities show that $C_{\phi}^{*} C_{\phi} / D_{\alpha}^{0}=F^{*} T_{\tau_{\phi, \alpha}} F / D_{\alpha}^{0}$. Since $F$ is an isometry, this suggests that the boundedness and compactness of $C_{\phi}$ can be described by the corresponding properties of $T_{\tau_{\phi, \alpha}}$.

In the main result we use the notion of integral averaging over basic sets in $\mathbb{D}$. There are several possible choices of basic sets, and we list three of them below.

1. Carleson disks $S(\xi, \delta)=\{z \in \mathbb{D} ;|z-\xi|<\delta\}$, where $0<\delta<1$ and $\xi \in \partial \mathbb{D}$.

2. Pseudohyperbolic disks: $D(a, \delta)=\left\{z \in \mathbb{D} ;\left|\frac{a-z}{1-\bar{a} z}\right|<\delta\right\}$ where $0<\delta<1$ and $a \in \mathbb{D}$.

3. Euclidean disks $D_{\delta}(a)=\{z \in \mathbb{D} ;|z-a|<\delta(1-|a|)\}$, where $0<\delta<1$ and $a \in \mathbb{D}$.

These sets all decompose $\mathbb{D}$ in similar way (for details see [3]). They are all equivalent with respect to their weighted area measure in the following sense:

- If $B$ is one of the basic sets 1 to 3 with fixed $\delta$, and if $z_{0}$ is either the center of mass in 1 and 2 , or the point $a$ from 3 , then, for $\alpha>-1, m_{\alpha}(B) \sim$ $\left(1-\left|z_{0}\right|\right)^{\alpha+2}$.

For details see [2] and [3].

\section{BOUNDEDNESS AND COMPACTNESS}

For a proof of the main result we still need to resolve in details two more obstacles: one is the boundedness and compactness criteria for Toeplitz operators on weighted Bergman spaces, and the other is dropping the restriction that the inducing function $\phi$ maps 0 to 0 . We shall do that with the aid of the following two lemmas. The first one is a special case of a more general result from [7].

Lemma 3.1 (Oleinik 1978, [7]). Let $\alpha>-1, D_{\frac{1}{4}}(a)=\left\{z \in \mathbb{D} ;|z-a|<\frac{1}{4}(1-|a|)\right\}$, and let $\tau_{\phi, \alpha}$ be the determining function for $\phi$ in the weighted Dirichlet space $D_{\alpha}$. Then

(i) The Toeplitz operator $T_{\tau_{\phi, \alpha}}$ is bounded on $A_{\alpha}$ if and only if

$$
\sup _{a \in \mathbb{D}} \frac{1}{(1-|a|)^{\alpha+2}} \int_{D_{\frac{1}{4}}(a)} \tau_{\phi, \alpha}(z) d m_{\alpha}(z)<\infty .
$$

(ii) The Toeplitz operator $T_{\tau_{\phi, \alpha}}$ is compact if and only if

$$
\lim _{r \mapsto 0} \sup _{(1-|a|)<r} \frac{1}{(1-|a|)^{\alpha+2}} \int_{D_{\frac{1}{4}}(a)} \tau_{\phi, \alpha}(z) d m_{\alpha}(z)=0 .
$$


Lemma 3.2. Let $\phi(0)=b \neq 0$ and let $\psi_{b}(z)=\frac{b-z}{1-\bar{b} z}$. If $\psi=\psi_{b} \circ \phi$, then

$$
\frac{1}{(1-|a|)^{\alpha+2}} \int_{D(a, \delta)} \tau_{\phi, \alpha}(z) d m_{\alpha}(z) \sim \frac{1}{(1-|c|)^{\alpha+2}} \int_{D(c, \delta)} \tau_{\psi, \alpha}(z) d m_{\alpha}(z),
$$

where $D(a, \delta)$ and $D(c, \delta)$ are pseudohyperbolic disks, and $c=\psi_{b}(a)$.

Proof. Since $\psi_{b}^{-1}=\psi_{b}$, we have that $\psi=\psi_{b} \circ \phi$ and $\phi=\psi_{b} \circ \psi$. Because of this symmetry it will be sufficient to prove that the expression on the left of the symbol $\sim$ is bounded by the product of a constant (depending only on $b$ ) and the expression on the right of the symbol $\sim$. Note that $\sum_{j}\left(1-\left|z_{j}(w)\right|\right)^{\alpha}=\sum_{j}\left(1-\left|u_{j}\left(\psi_{b}(w)\right)\right|\right)^{\alpha}$, where $z_{j}(w)$ are $\phi$ preimages of $w$ and $u_{j}\left(\psi_{b}(w)\right)$ are $\psi_{b} \circ \phi$ preimages of $\psi_{b}(w)$. Thus

$$
\tau_{\phi, \alpha}(w)(1-|w|)^{\alpha}=\tau_{\psi_{b} \circ \phi, \alpha}\left(\psi_{b}(w)\right)\left(1-\left|\psi_{b}(w)\right|\right)^{\alpha} .
$$

In addition, the pseudohyperbolic metric is disk-automorphism invariant and so, for a fixed $\delta>0, \psi_{b}(D(a, \delta))=D\left(\psi_{b}(a), \delta\right)$ for any $a \in \mathbb{D}$.

Using these two facts we obtain

$$
\begin{aligned}
& \frac{1}{(1-|a|)^{\alpha+2}} \int_{D(a, \delta)} \tau_{\psi, \alpha}(w)(1-|w|)^{\alpha} d m(w) \\
& =\frac{1}{(1-|a|)^{\alpha+2}} \int_{D(a, \delta)} \tau_{\psi_{b} \circ \phi, \alpha}\left(\psi_{b}(w)\right)\left(1-\left|\psi_{b}(w)\right|\right)^{\alpha} \cdot d m(w) \\
& \stackrel{\mathrm{C} . \mathrm{V}}{=} \frac{1}{(1-|a|)^{\alpha+2}} \int_{\psi_{b}(D(a, \delta))} \tau_{\psi, \alpha}(z)(1-|z|)^{\alpha} \cdot\left|\psi_{b}^{\prime}(z)\right|^{2} d m(z) \\
& =\left(\frac{1-\left|\psi_{b}(a)\right|}{1-|a|}\right)^{\alpha+2} \frac{1}{\left(1-\left|\psi_{b}(a)\right|\right)^{\alpha+2}} \int_{\psi_{b}(D(a, \delta))} \tau_{\psi, \alpha}(z)(1-|z|)^{\alpha}\left(\frac{1-|b|^{2}}{|1-\bar{b} z|^{2}}\right)^{2} d m(z) \\
& \leq(\operatorname{const}(b))^{\alpha+2} \cdot\left(\frac{1+|b|}{1-|b|}\right)^{2} \cdot \frac{1}{(1-|c|)^{\alpha+2}} \int_{D(c, \delta)} \tau_{\psi, \alpha}(z) d m(z) \\
& =\operatorname{const}(b, \alpha) \frac{1}{(1-|c|)^{\alpha+2}} \int_{D(c, \delta)} \tau_{\psi, \alpha}(z) d m(z),
\end{aligned}
$$

where C.V. stands for "change of the variable $\psi_{b}(w)=z$ ", and $c=\psi_{b}(a)$. The above inequality follows from the fact that for the disk automorphism $\psi_{b}(z)$ we have that

$$
\begin{gathered}
1-\left|\psi_{b}(a)\right|=\frac{1}{\left|1+\psi_{b}(a)\right|} \cdot \frac{\left(1-|a|^{2}\right)(1-|b|)^{2}}{|1-\bar{b} a|^{2}} \leq \frac{2}{(1-|b|)^{2}}(1-|a|) \\
=\operatorname{const}(b) \cdot(1-|a|),
\end{gathered}
$$

and that

$$
\frac{1-|b|^{2}}{|1-\bar{b} z|} \leq \frac{1+|b|}{1-|b|}
$$

We are now ready to prove the main theorem as stated in the Introduction. We use pseudohyperbolic disks $D(a, \delta)$ as basic sets. Note that any of the other two kinds of basic sets could have been used as well.

Proof of Theorem 1. Suppose first that $\phi$ in $D_{\alpha}$ is such that $\phi(0)=0$.

Let us recall the connection between the composition operator $C_{\phi}$ on $D_{\alpha}$ and the Toeplitz operator $T_{\tau_{\phi, \alpha}}$ on $A_{\alpha}$ from section 1. 
- Since $T_{\tau_{\phi, \alpha}}$ is in $L^{1}\left(d m_{\alpha}\right)$, the Toeplitz operator $T_{\tau_{\phi, \alpha}}$ is well defined.

- For every polynomial $f$ and $g$ in $D_{\alpha}$ that maps 0 to 0 , we have that

$$
\left\langle C_{\phi}^{*} C_{\phi} f, g\right\rangle_{D_{\alpha}}=\left\langle T_{\tau_{\phi, \alpha}} f^{\prime}, g^{\prime}\right\rangle_{A_{\alpha}} .
$$

In other words, $C_{\phi}$ is bounded (or compact) on $D_{\alpha}$ (or, more precisely, on the invariant subspace $D_{\alpha}^{0}$ ) if and only if $T_{\tau_{\phi, \alpha}}$ is bounded (or compact) on $A_{\alpha}$.

Using Lemma 3.1 and the equivalence of basic sets we conclude that $C_{\phi}$ is bounded (or compact) in $D_{\alpha}$ if and only if the corresponding $\sup$ (or $\lim$ as $|a| \mapsto 1$ ) of the expression

$$
\frac{1}{(1-|a|)^{\alpha+2}} \int_{D(a, \delta)} \tau_{\phi, \alpha} d m_{\alpha}(z)
$$

is satisfied for some $\delta>0$.

To get the theorem (for the case $\phi(0)=0$ ) we only have to add the following comment: for every $z \in D(a, \delta)$, we have that $1-|z| \sim 1-|a|$ (see [2]). So

$$
\begin{gathered}
\frac{1}{(1-|a|)^{\alpha+2}} \int_{D(a, \delta)} \tau_{\phi, \alpha}(z) d m_{\alpha}(z) \\
\sim \frac{1}{(1-|a|)^{\alpha+2}}(1-|a|)^{\alpha} \int_{D(a, \delta)} \tau_{\phi, \alpha}(z) d m(z) \\
=\frac{1}{(1-|a|)^{2}} \int_{D(a, \delta)} \tau_{\phi, \alpha}(z) d m(z) \\
=\frac{1}{m(D(a, \delta))} \int_{D(a, \delta)} \tau_{\phi, \alpha}(z) d m(z) .
\end{gathered}
$$

Now, if $\phi(0)=b \neq 0$, then, by Lemma 3.2 and the preceding comments for the function $\psi(z)=\psi_{b}(\phi(z))$, we again derive the same necessary and sufficient condition on the determining function $\tau_{\phi, \alpha}$.

\section{Comments And EXAMPles}

In this section we explain the connection between the main result and the known results on boundedness and compactness of composition operators on weighted Bergman and Dirichlet spaces. We show in the examples below that, even though our characterization in Theorem 1 appears too abstract, it is a rather powerful geometric tool, easily applicable to some concrete functions and spaces.

In the main theorem we consider $\alpha$ in the interval $(-1,1)$. If $\alpha>1$ the weighted Dirichlet spaces are the weighted Bergman spaces $A_{\alpha-2}$, while $\alpha=1$ yields the classical Hardy space $H^{2}=A_{-1}$. The same proof as above gives:

- $C_{\phi}$ is bounded (or compact) on $A_{\alpha}, \alpha \geq-1$, if and only if there exists $\delta$, $0<\delta<1$, such that

$$
\begin{gathered}
\sup _{a \in \mathbb{D}} \frac{1}{m(D(a, \delta))} \int_{D(a, \delta)} \tau_{\phi, \alpha+2}(w) d m(w)<\infty \\
\text { (or } \lim _{a \mapsto \partial D} \frac{1}{m(D(a, \delta))} \int_{D(a, \delta)} \tau_{\phi, \alpha+2}(w) d m(w)=0 . \text { ) }
\end{gathered}
$$

How does this relate to, for example, the well known result that every composition operator on weighted Bergman spaces and on the classical Hardy space is bounded? 
First of all, since $\alpha+2 \geq 1$, the determining function $\tau_{\phi, \alpha+2}$ has the sub-mean value property (for $|a|$ close to 1 ), i.e.

$$
\tau_{\phi, \alpha+2}(a) \leq \frac{\text { const }}{m(D(a, r))} \int_{D(a, r)} \tau_{\phi, \alpha+2}(w) d m(w) .
$$

Hence, the condition that $\tau_{\phi, \alpha+2}(a)$ is bounded (for $|a|$ close to 1 ) is both a sufficient and necessary condition for boundedness of $C_{\phi}$ on $A_{\alpha}$.

Secondly, for $\alpha+2 \geq 1$,

$$
\tau_{\phi, \alpha+2}(a) \leq\left(\tau_{\phi, 1}(a)\right)^{\alpha+2} \leq\left(\frac{1+|\phi(0)|}{1-|\phi(0)|}\right)^{\alpha+2}
$$

for $|a|$ close to 1, where the last inequality is (essentially) the well known Littlewood's inequality. For details see [9]. So, every composition operator $C_{\phi}$ is bounded on $A_{\alpha}$.

The sub-mean value property of $\tau_{\phi, \alpha}, \alpha \geq 1$, can also be used to get a connection between the upper lim condition over the integral averages and the well known limsup condition on $\tau_{\phi, \alpha}$ for compactness of $C_{\phi}$ from [9].

For $\mathbf{0}<\boldsymbol{\alpha}<\mathbf{1}$, the multiplicity of $\phi$ plays a very important rôle. If $\phi$ is of bounded multiplicity and if $\phi$ is in $D_{\alpha}, \alpha>0$, then $C_{\phi}$ is bounded on $D_{\alpha}$ (see [9]). But if $\phi$ is of a bounded multiplicity $M$ and if $\phi(0)=0$, then, by the Schwartz lemma, $|\phi(z)|<|z|$ for all $z \in \mathbb{D}$. Hence, in that case

$$
\frac{1-\left|z_{j}(w)\right|}{1-|w|}=\frac{1-\left|z_{j}(w)\right|}{1-\left|\phi\left(z_{j}(w)\right)\right|}<1
$$

and we have that $\tau_{\phi, \alpha}(w) \leq M$ for $\alpha>0$. All of the Dirichlet spaces are diskautomorphism invariant, and so, it follows from our main theorem that $C_{\phi}$ is bounded on $D_{\alpha}, \alpha>0$, for every $\phi$ in $D_{\alpha}$ with bounded multiplicity.

Composition operators of inner functions are of special interest. If $\phi$ is inner and has no angular derivatives on the unit circle, then $C_{\phi}$ cannot be bounded on $D_{\alpha}, \alpha<1$ (see [9]). Examples of functions $\phi$ for which the boundedness of $C_{\phi}$ in $D_{\alpha}$ is not yet explicitly resolved are examples of inner functions with unbounded multiplicity that have an angular derivative at at least one point of the unit circle. Will these functions still induce unbounded composition operators on $D_{\alpha}, \alpha<1$ ?

If $\alpha<0$, then only inner functions in $D_{\alpha}$ are finite Blashke products. If $\alpha \leq \frac{1}{2}$, no singular inner function is in $D_{\alpha}$. But if $\alpha>\frac{1}{2}$, then the spaces $D_{\alpha}$ are big enough to include a variety of inner functions.

For example, the singular inner function $\phi(z)=\exp \left(\frac{1+z}{1-z}\right)$ is in $D_{\alpha}, \alpha>\frac{1}{2}$. It is a function with unbounded multiplicity, and has angular derivatives everywhere on $\partial \mathbb{D}$, except at the point 1 . We shall use our main theorem (Theorem 1) and show that $C_{\phi}$ is bounded on $D_{\alpha}$, whenever $\alpha>\frac{1}{2}$.

Example 1. Let $\phi(z)=\exp \left(\frac{z+1}{z-1}\right)$. Then $\phi$ maps $\mathbb{D}$ into itself and $\phi \in D_{\alpha}, \alpha>\frac{1}{2}$ (see [6]). We shall prove that $\lim \sup _{|w| \mapsto 1} \tau_{\phi, \alpha}(w)<\infty$.

Let $\left\{w_{n}\right\}$ be such that $\lim _{n \mapsto \infty} \tau_{\phi, \alpha}\left(w_{n}\right)=\lim \sup _{|w| \mapsto 1} \tau_{\phi, \alpha}(w)$. Then $w_{n}=$ $e^{u_{n}} e^{i v_{n}}$, where $u_{n}<0,0 \leq v_{n}<2 \pi$, and $u_{n} \mapsto 0$ as $n \mapsto \infty$. We also have that

$$
\phi^{-1}\left(w_{n}\right)=\left\{z_{j}(n) ; z_{j}(n)=\frac{u_{n}+i\left(v_{n}+z_{j} \pi\right)+1}{u_{n}+i\left(v_{n}+z_{j} \pi\right)-1}, i \in \mathbb{Z}\right\} .
$$


We want to show that $\tau_{\phi, \alpha}\left(w_{n}\right)$ is bounded for all $n$. Recall that

$$
\tau_{\phi, \alpha}\left(w_{n}\right)=\sum_{j}\left(\frac{1-\left|z_{j}(n)\right|}{1-\left|w_{n}\right|}\right)^{\alpha} \leq \sum_{j}\left(\frac{1-\left|z_{j}(n)\right|^{2}}{1-\left|w_{n}\right|}\right)^{\alpha} .
$$

It is not hard to see that the vertical lines $x=u_{n}$ in the left half plane are images of orocycles $C_{n}=\left\{z ; \frac{1-|z|^{2}}{|1-z|^{2}}=u_{n}\right\}$ under the map $\frac{z+1}{z-1}$. The points $u_{n}+i\left(v_{n}+z_{j} \pi\right)$ are on the horizontal lines $y=v_{n}+2 j \pi$, and the latter are images of the circles $S_{n j}=\left\{z ;\left|z-\left(1-i \frac{1}{v_{n}+2 \pi j}\right)\right|=\left|\frac{-1}{v_{n}+2 \pi j}\right|\right\}$ under the map $\frac{z+1}{z-1}$.

Note that $C_{n}$ 's are circles internally tangent to $\partial \mathbb{D}$ at the point 1 , while $S_{n j}$ 's are circles tangent to the real axis at the point 1, above (or below) the real axis if $j$ is negative (or positive, respectively). Both $C_{n}$ and $S_{n j}$ converge to 1 as $n \mapsto \infty$, and $S_{n j}$ converges to 1 as $j \mapsto \infty$, for every $n$.

Combining the formulas for $C_{n}$ and $S_{n j}$, we get that

$$
1-\left|z_{j}(n)\right|^{2}=\frac{-4 u_{n}}{\left(u_{n}-1\right)^{2}+\left(v_{n}+2 j \pi\right)^{2}},
$$

where $u_{n}<0$, and so

$$
\tau_{\phi, \alpha}\left(w_{n}\right) \leq\left(\frac{1}{1-e^{u_{n}}}\right)^{\alpha} 4^{\alpha}\left(-u_{n}\right)^{\alpha} \sum_{j}\left(\frac{1}{\left(u_{n}-1\right)^{2}+\left(v_{n}+2 j \pi\right)^{2}}\right)^{\alpha} .
$$

As $n \rightarrow \infty$, we have,

$$
\begin{gathered}
\lim _{n \mapsto \infty} \tau_{\phi, \alpha}\left(w_{n}\right)<4^{\alpha} \lim _{n \mapsto \infty}\left(\frac{-u_{n}}{1-e^{u_{n}}}\right)^{\alpha} \sum_{j} \frac{1}{(2 j \pi)^{2 \alpha}} \\
=4^{\alpha} M_{\alpha} \lim _{x \mapsto 0}\left(\frac{-x}{1-e^{x}}\right)^{\alpha}=4^{\alpha} M_{\alpha},
\end{gathered}
$$

where the sum converges since $2 \alpha>1$. So, $\lim _{\sup } \sup _{|w|} \tau_{\phi, \alpha}(w)<\infty$, and by Theorem $1, C_{\phi}$ is bounded on $D_{\alpha}, \alpha>\frac{1}{2}$.

It has been proven in [5] that if $C_{\phi}$ is bounded on $D_{\gamma}$ and if $0 \leq \gamma<\alpha<1$, then $C_{\phi}$ is bounded on $D_{\alpha}$. This result follows from Theorem 1 and the fact that $\tau_{\phi, \gamma}(w) \geq \tau_{\phi, \alpha}(w)$ for all $w \in \mathbb{D}$ and $\phi$ with $\phi(0)=0$.

It is not known if $\tau_{\phi, \alpha}$ for $0<\alpha<1$ satisfies the sub-mean value property, i.e. if the integral averaging approach could be improved by a pointwise characterization.

For $\boldsymbol{\alpha}=\mathbf{0}$ the following example shows that, in general, $\tau_{\phi, 0}$ does not have the sub-mean value property and that the characterization from Theorem 1 is the best possible. The example is a slight modification of an example constructed (for another purpose) by Joel Shapiro in [11].

Recall that for $\alpha=0, \tau_{\phi, 0}(w)=n_{\phi}(w)$ (the multiplicity of $\phi$ at $w$ ). Using Carleson squares for basic sets the main theorem gets the following form, which is already known ([11]).

- For $\phi$ in the Dirichlet space $\mathcal{D}$, the composition operator $C_{\phi}$ is bounded (or compact) if and only if there exists a constant $C>0$ such that for all $\xi \in \partial \mathbb{D}$,

$$
\begin{gathered}
\int_{S(\xi, \delta)} n_{\phi}(w) d m(w) \leq c \delta^{2}, \text { for all } \delta, 0<\delta<1 \\
\left(\text { or } \lim _{\delta \mapsto 0} \frac{1}{\delta^{2}} \int_{S(\xi, \delta)} n_{\phi}(w) d m(w)=0\right) .
\end{gathered}
$$




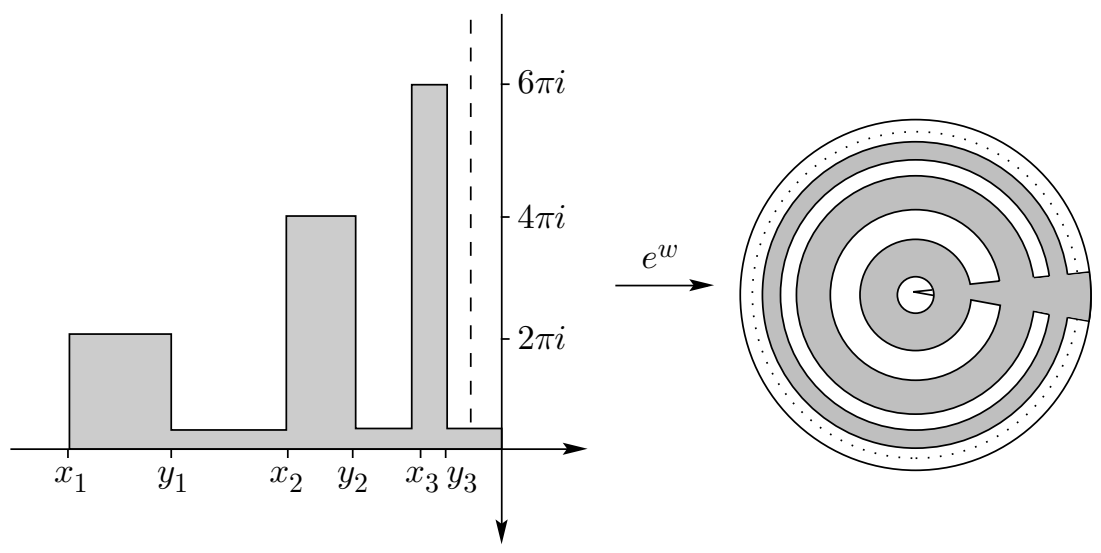

FiguRE 1

Example 2. Let $\Omega$ be the open simply connected domain made from the rectangles $P_{j}$ with width interval $\left(x_{j}=\log \left(1-\frac{1}{j+1}\right), y_{j}\right)$, where $y_{j}<\log \left(1-\frac{1}{j+2}\right)$ and with height interval $(0, j 2 \pi i)$. All of the rectangles are connected at their base with a thin horizontal strip (see Figure 1 ). Let $\phi_{1}$ be the Riemann map from $\mathbb{D}$ onto $\Omega$, and let $\phi(z)=\exp \left(\phi_{1}(z)\right)$. Then $\phi$ maps $\mathbb{D}$ onto $\exp (\Omega)$, where $\exp (\Omega)$ is made out of the rings $R_{j}$ (the images of the rectangles $P_{j}$ ). The rings are included in $\mathbb{D}$ (see Figure 1), and on each of $R_{j}$ the multiplicity $n_{\phi}(w)$ of $\phi$ is equal to $j$.

The sequence $\left\{y_{j}\right\}$ determines the area of the rings $R_{j}$. Choose $y_{j}$ 's that make the rectangles $P_{j}$ so thin that the area of $R_{j}$ is of size $m\left(R_{j}\right)=\frac{1}{j^{2^{j}}}$.

We will show that the operator $C_{\phi}$ is bounded on $\mathcal{D}$ even though

$$
\lim \sup _{|w| \mapsto 1} \tau_{\phi, 0}(w)=\lim \sup _{|w| \mapsto 1} n_{\phi}(w)=\infty
$$

(the latter equality is true since the multiplicity of $\phi$ on $R_{j}$ is $j$ ).

We need to estimate the integral average of $n_{\phi}(w)$ by using Carleson squares as basic sets. Note that this estimation will not depend on the point $\xi$, except for $\xi$ close to 1 . But for $\xi$ close to 1, we only need to add portions of $\phi(\mathbb{D})$ on which the multiplicity of $\phi$ is 1 , and that will not alter the finitness.

For $\delta$ such that $\frac{1}{N+1} \leq \delta<N$, the Carleson square $S(\xi, \delta)$ intersects with only a $2 \delta$-fraction of each of the rings $R_{j}, j \geq N$, i.e. $m\left(S(\xi, \delta) \cap R_{j}\right)=2 \delta \cdot m\left(R_{j}\right)$. If $j<N$, then $S(\xi, \delta) \cap R_{j}=\emptyset$.

Thus, for $\frac{1}{N+1} \leq \delta<N$, we have

$$
\begin{gathered}
\int_{S(\xi, \delta)} n_{\phi}(w) d m(w)=\sum_{j \geq N} j \cdot m\left(S(\xi, \delta) \cap R_{j}\right) \\
=\sum_{j \geq N} j \cdot 2 \delta \cdot \frac{1}{j 2^{j}}=2 \delta \frac{1}{2^{N}} \leq \delta^{2}
\end{gathered}
$$

(whenever $N \geq 2$ ), and so $C_{\phi}$ is bounded on $\mathcal{D}$.

Note that the function $\phi$ is in $\mathcal{D}$ since $\sum_{j=1}^{\infty} j \cdot \frac{1}{j 2^{j}}=1<\infty$. 
Choosing the rings $R_{j}$ to be this thin, we have actually made $C_{\phi}$ to be compact on $\mathcal{D}$ (even though $\lim \sup _{|m| \mapsto 1} \tau_{\phi, 0}(w)=\infty$ ), since for $\frac{1}{N+1} \leq \delta<\frac{1}{N}$

$$
\frac{1}{\delta^{2}} \int_{S(\xi, \delta)} n_{\phi}(w) d m(w)=\frac{1}{\delta^{2}} \frac{\delta}{2^{N}}=\frac{1}{\delta \cdot 2^{N}} \leq \frac{N+1}{2^{N}} \mapsto 0
$$

as $N \mapsto \infty$, that is, as $\delta \mapsto 0$.

For $-\mathbf{1}<\boldsymbol{\alpha}<\mathbf{0}$, the functions in $D_{\alpha}$ are all continuous on the unit circle. It is known that if $C_{\phi}$ is bounded on $D_{\alpha}$, then $\phi$ must have an angular derivative at every point $\xi$ with $|\phi(\xi)|=1$, and that $C_{\phi}$ is compact on $D_{\alpha}$ if and only if $\phi \in D_{\alpha}$ and $\|\phi\|_{\infty}<1$ (see [1] and [8]).

If $-1<\gamma<\alpha<0$, then $C_{\phi}$ bounded on $D_{\gamma}$ implies $C_{\phi}$ bounded in $D_{\alpha}([5])$. This result and the fact that the compactness of $C_{\phi}$ on $D_{\alpha}$ is a consequence of the compactness of $C_{\phi}$ on $D_{\gamma}$ again follow easily from Theorem 1 , since $\phi(0)=0$ implies $\tau_{\phi, \gamma}(w) \geq \tau_{\phi, \alpha}(w)$ for all $w \in \mathbb{D}$.

For this range of $\alpha$ 's, the multiplicity of $\phi$ is not essential anymore. For example, if $\phi$ is univalent function in $D_{\alpha},|\phi(\xi)|=1$ and $\phi$ has no angular derivative at $\xi$, then $C_{\phi}$ is unbounded on $D_{\alpha}$. The predominant notion in this case is the angular derivative. The proof given in [1], page 186, does not give us explicit, geometric reasons why this happens. Theorem 1 on the other hand provides a geometrical insight into what goes wrong in this case, as follows.

Whenever $|\phi(\xi)|=1$ and $\phi$ has no angular derivative at $\xi$, then $\tau_{\phi, \alpha}(\phi(z))$ is unbounded in a neighborhood of $\xi$ even though $\phi$ is univalent. This is true since

$$
\tau_{\phi, \alpha}(\phi(z))=\left(\frac{1-|\phi(z)|}{1-|z|}\right)^{-\alpha}
$$

and for $z$ close to $\xi$ the right-hand side is close to $\left|\phi^{\prime}(\xi)\right|^{-\alpha}$, where $-\alpha>0$.

If $\phi$ is in $D_{\alpha},-1<\alpha<0$, then $\phi(\mathbb{D})$ near $\phi(\xi)$ must include a sector with a vertex at $\phi(\xi)$ and an angular opening $\theta$. This follows from the fact that $D_{\alpha} \subset \operatorname{Lip}\left(-\frac{\alpha}{2}\right)$ (Lipschitz functions on $\overline{\mathbb{D}}$ with index $-\frac{\alpha}{2}$ ) and the property that images of univalent Lipschitz functions cannot have zero-angle cusps. For details see for example [12].

Thus, there exists a constant $C$ (depending only on $\theta$ ) such that

$$
m(\phi(\mathbb{D}) \cap S(\xi, \delta)) \geq C \cdot m(S(\xi, \delta))
$$

for small enough $\delta$. Hence, for every $N>0$, there exists $\delta>0$ such that

$$
\frac{1}{m(S(\xi, \delta))} \int_{S(\xi, \delta)} \tau_{\phi, \alpha}(w) d m(w) \geq N \cdot C
$$

and by Theorem $1, C_{\phi}$ is unbounded in $D_{\alpha}$.

Using Theorem 1 it is also easy to see geometrically why $\|\phi\|_{\infty}<1$ is a necessary condition for the compactness of $C_{\phi}$ on $D_{\alpha},-1<\alpha<0$. Note that if $C_{\phi}$ is bounded in $D_{\alpha}$ and $|\phi(\xi)|=1$, then $\phi$ must have an angular derivative at $\xi$; i.e. the arc from the unit circle containing $\xi$ is mapped by $\phi$ into a curve that is tangent to the unit circle at $\phi(\xi)$. The main geometrical idea is thus illustrated by the following example.

Example 3. Let $\phi$ be the univalent map from $D_{\alpha},-1>\alpha>0$, that maps the unit disc onto an internally tangent disc such that $\phi(1)=1$. Whenever $w$ from $\phi(\mathbb{D})$ is close to 1 , we have that $\tau_{\phi, \alpha}(w)$ is close to $\left|\phi^{\prime}(1)\right|^{-\alpha}$. Let $D_{\delta}(a)$ be the Euclidean $\operatorname{disc}\{z \in \mathbb{D} ;|z-a|<\delta(1-|a|)\}$. It is not hard to see that for every $\delta$, 
$0<\delta<1$, there exists $a \in \mathbb{D}, a$ close enough to 1 , such that $D_{\delta}(a) \subset \phi(\mathbb{D})$. But then

$$
\tau_{\phi, \alpha}(w) \geq \frac{\left|\phi^{\prime}(1)\right|^{-\alpha}}{2}
$$

for all $w \in D_{\delta}(a)$. Hence, for every $\delta$,

$$
\limsup _{|a| \mapsto 1} \frac{1}{m\left(D_{\delta(a)}\right)} \int_{D_{\delta}(a)} \tau_{\phi, \alpha}(w) d m(w) \geq \frac{\left|\phi^{\prime}(1)\right|^{-\alpha}}{2}>0
$$

and by Theorem $1, C_{\phi}$ cannot be compact on $D_{\alpha}$.

\section{REFERENCES}

1. C.C.Cowen and B.D.MacCluer, Composition Operators on Spaces of Analytic Functions, CRC Press, Boca Raton, 1995. CMP 96:14

2. D.H.Luecking, Inequalities on Bergman spaces, Illinois J.Math. 25 (1981), 1-11. MR 82e:30072

3. D.H.Luecking, Trace ideal criteria for Toeplitz operators, J. Functional Analysis 73 (1987), 345-368. MR 88m:47046

4. D.H.Luecking and K.Zhu, Composition operators belonging to the Schatten ideals, American J.Math. 114 (1992), 1127-1145. MR 93i:47032

5. B.D. MacCluer and J.H. Shapiro, Angular derivatives and compact composition operators on the Hardy and Bergman spaces, Canadian J.Math. 38 (1986), 878-906. MR 87h:47048

6. D.J. Newman, H.S. Shapiro, Embedding theorems for weighted classes of harmonic and analytic functions, Michigan Math. J. 9 (1962), 249-255

7. V.L.Oleinik, Embedding theorems for weighted classes of harmonic and analytic functions, Journal of Soviet Mathematics 9 (1978), 228-243.

8. J.H. Shapiro, Compact composition operators on spaces of boundary regular holomorphic functions, Proc.Amer.Math.Soc 100 (1987), 49-57. MR 88c:47059

9. J.H. Shapiro, The essential norm of a composition operator, Annals Math. 125 (1987), 375404. MR 88c: 47058

10. J.H. Shapiro, Composition Operators and Classical Function Theory, Springer-Verlag, New York, 1993. MR 94k:47049

11. J.H. Shapiro, Private communication

12. W. Smith and D.A. Stegenga, A geometric characterization of Holder domains, J.London Math.Soc (2) 35, (1987), 471-480. MR 88f:30011

13. N. Zorboska, Composition operators with closed range, Trans. Amer. Math. Soc. 344 (1994), 791-801. MR 94k:47050

Department of Mathematics and Astronomy, University of Manitoba, Winnipeg, ManITOBA, CANADA R3T 2N2

E-mail address: zorbosk@ccu.umanitoba.ca 